\title{
Study on Forecasting Method of Wheel-Rail Vertical Force by Frameload
}

\author{
$\mathrm{Ze} \mathrm{Li}$ \\ Chongqing Vocational College of Transportation, Chongqing, China
}

Keywords: Wheel-rail force, Rigid-flexible coupling, Pearson coefficient, Relevance

\begin{abstract}
Wheel-rail interaction force is the basis of studying wheel-rail relationship and one of the important elements of evaluating vehicle operation safety. At present, the measurement of wheel-rail force is still the focus and difficulty in the field of railway vehicle research, however, line test using dynamometer bogie frame has a high leve. Therefore, in this paper, combined with rigid-flexible coupling multi-body dynamics, the whole vehicle dynamics model of high-speed train is established. Vertical force of wheel and rail and vertical load of frame under different working conditions are obtained by simulation. Pearson coefficient is used to analyze the correlation between them and extract the correlation characteristics. It is proposed that vertical load of frame be used to advance the vertical force of wheel and rail.
\end{abstract}

\section{Introduction}

Nowadays, the operation speed of Fuxing EMU has reached $350 \mathrm{~km} / \mathrm{h}$. The high-speed increase of train speed will also lead to the aggravation of the action force between wheel and rail, which will inevitably endanger the stability, comfort and safety of vehicle operation ${ }^{[1]}$. At present, the measurement of wheel-rail force is still the key and difficult point in the field of railway vehicle research, and the use of force measuring frame for line testing has a high level. When the vehicle is running, the force between wheel and rail is transmitted to the frame in the form of vibration through the suspension device, which causes the load change of the frame. It can be seen that there is a certain relationship between wheel-rail force and frame load. If we can predict wheel-rail force by analyzing the correlation between wheel-rail force and frame load, it will have a certain economic value.

In this paper, combined with rigid-flexible coupling multi-body dynamics, the whole vehicle dynamics model of high-speed train is established. Vertical force of wheel and rail and vertical load of frame under different working conditions are obtained by simulation. The correlation between them is analyzed by Pearson coefficient and the correlation characteristics are extracted. The vertical force of wheel and rail by vertical load of frame is proposed. The method of forecasting.

\section{Establishment of Rigid-Flexible Coupling Dynamic Model for High Speed Emu}

\subsection{Bogie Frame Structure}

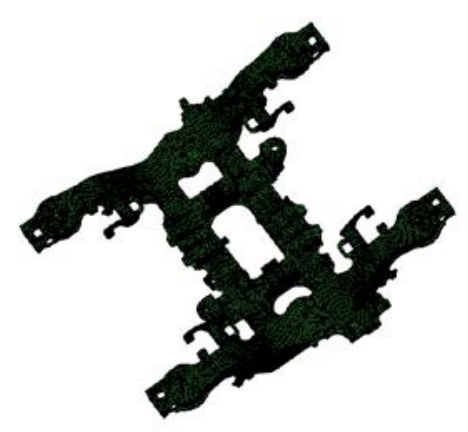

Fig.1 Finite Element Model of Motor Vehicle Bogie Frame

In order to deal with the elasticity of bogie frame, the elastomer is discretized, the bogie frame is discretized into a finite number of elements, and nodes are set to connect the elements, so that the 
relevant parameters of the elements are continuous and constitute a set, as shown in Figure 1. Because it is necessary to import the software of flexible body into SIMPACK for rigid-flexible coupling calculation, we need to extract finite element shape *. CDB file and sub-structure *. sub file ${ }^{[2,3]}$ in ANSYS.

\subsection{Establishment of Vehicle Model}

Modeling in SIMPACK, users can set parameters by calling corresponding modules, including stiffness, center of gravity, moment of inertia, mass and damping of the body, create corresponding force elements and constraints according to the needs of elastic elements, thus completing the creation of multi-body dynamic model, and obtain dynamic simulation results through integral operation $^{[4]}$.

(1) When the wheelset is established, the articulated connection of No. 7 with six degrees of freedom is adopted.

(2) When establishing wheel-rail contact, the Huertz contact theory and Kalker non-linear creep theory defaulted by SIMPACK are selected, and LMA tread and UIC60 rail are selected. The geometric diagram of wheel-rail contact is as follows.

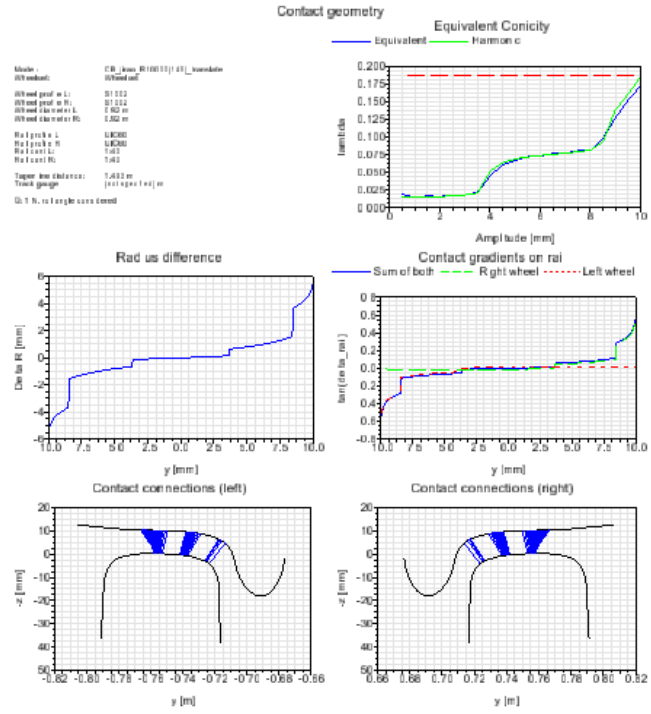

Fig.2 Geometric Relationship of Wheel-Rail Contact

(3) Build a bogie, import the flexible body frame into SIMPCK, then use the sub-structure interface to import the wheelset, and establish constraints and connections according to the actual vehicle conditions. In addition, the actual model should be equivalent to the corresponding mechanical model, such as the spring-damper parallel element simulation for a suspension system.

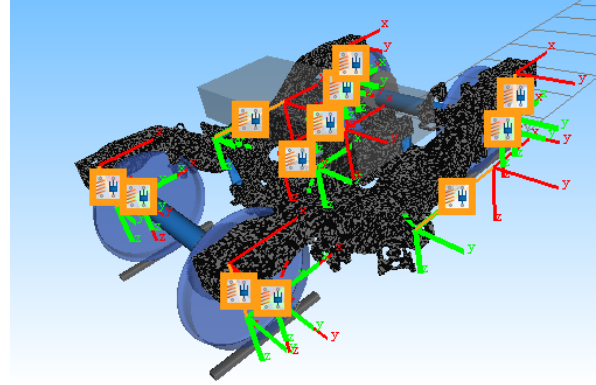

Fig.3 Bogie Model

(4) The whole vehicle model is established, the bogie is imported by substructure interface, and the secondary air spring is replaced by the force element of spring-damp parallel connection. The whole vehicle model is as follows: 


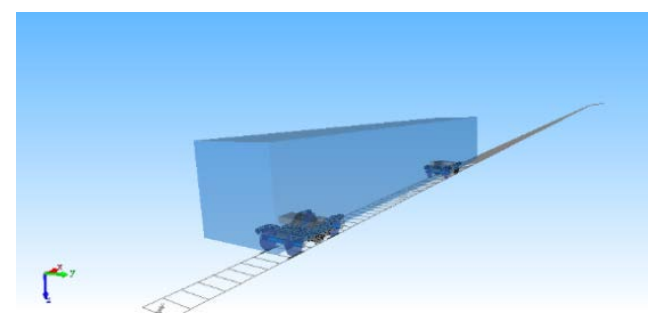

Fig.4 Dynamic Model of Rigid-Flexible Coupled Vehicle System

\section{Vertical Correlation Analysis}

\subsection{Analysis Method}

Relevance analysis refers to the correlation analysis of two or more variables to measure the degree of correlation between variables. Relevance refers to whether there is consistency in the way two variables change. If the two variables show the same fluctuation, if they rise or fall at the same time, they are considered to be highly correlated; otherwise, they are considered to be weakly correlated. The correlation coefficient reflects the correlation intensity between the two variables. Pearson coefficient is commonly used in the calculation method. It is expressed by the product ratio of covariance between two variables and standard deviation between two variables. The calculation formula is as follows:

$$
\begin{array}{r}
r=\frac{\sigma_{\mathrm{xy}}^{2}}{\sigma_{\mathrm{x}} \sigma_{\mathrm{y}}}=\frac{\sum(\mathrm{x}-\overline{\mathrm{x}})(\mathrm{y}-\overline{\mathrm{y}})}{\sqrt{\sum(\mathrm{x}-\overline{\mathrm{x}})^{2}(\mathrm{y}-\overline{\mathrm{y}})^{2}}} \\
=\frac{\sqrt{\overline{\mathrm{xy}}-\overline{\mathrm{x}} \mathrm{g} \overline{\mathrm{y}}}}{\sqrt{\left(\overline{\mathrm{x}}^{2}-\overline{\mathrm{x}}^{2}\right) \mathrm{g}\left(\overline{\mathrm{y}}^{2}-\overline{\mathrm{y}}^{2}\right)}} \\
=\frac{n \sum x y-\sum x \sum y}{\sqrt{n \sum x^{2}-\left(\sum x\right)^{2}} \sqrt{n \sum y^{2}-\left(\sum y\right)^{2}}}
\end{array}
$$

The range of correlation coefficient is: $-1 \leq \mathrm{r} \leq 1$.Negative values indicate negative correlation and positive values indicate positive correlation. Using numerical values to measure the degree of closeness of variables is usually shown as follows:

When $|\mathrm{r}|=0$ is completely unrelated, $0<|\mathrm{r}|<0.3$ is weak correlation, when $0.3<|\mathrm{r}|<0.5$ is low correlation, when $0.5<|\mathrm{r}|<0.8$ is significant correlation, when $0.8<|\mathrm{r}|<1$ is highly correlation, and when $|\mathrm{r}|=1$ is complete correlation.

\subsection{Time Domain Analysis}

The correlation between the vertical load of the frame and the vertical force of the wheel and rail at $60,120,160,200,280$ and $300 \mathrm{~km} / \mathrm{h}$ speeds is analyzed respectively. For the analysis of the vertical correlation in time domain, we extract the data of the semi-car body. The results of the correlation at each speed are as follows:

Table 1 Coefficient of Correlation at Different Velocities

\begin{tabular}{|l|l|l|l|l|l|l|}
\hline speed & 60 & 120 & 160 & 200 & 280 & 300 \\
\hline coefficient & 0.262 & 0.094 & 0.052 & 0.037 & 0.153 & 0.178 \\
\hline
\end{tabular}

From Table 1, it can be seen that the correlation coefficients between wheel-rail vertical force and frame vertical load at different speeds are greater than 0 , but less than 0.3 , which is a weak correlation. Taking the correlation between the two variables directly, we neglect the influence of phase difference caused by the transmission of different frequency components of wheel-rail vertical force to the frame. 


\subsection{Frequency Domain Analysis}

Spectrum analysis of wheel-rail vertical force and frame vertical load at different speed levels of low, medium and high is carried out, as shown in Fig. 5:

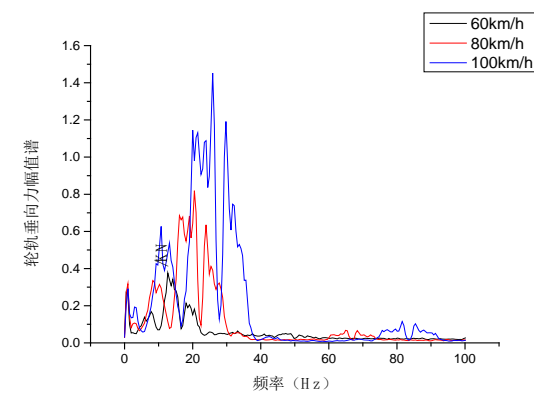

(a)Low Speed Wheel-Rail Force

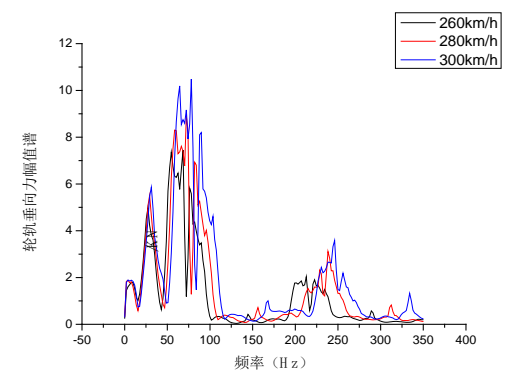

(b)Medium and High Speed Wheel/Rail Force

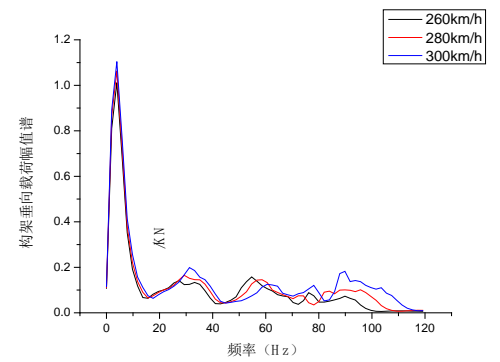

(c)High Speed Frame Load

Fig.5 Spectrum Analysis

It is found that with the increase of train speed, the influence of high frequency components on wheel-rail vertical force is more and more significant, but the high frequency components are obviously restrained when they are transmitted to the frame due to the vibration reduction of the primary suspension system. When the train runs at medium and low speed, the transmission efficiency between wheel-rail vertical force and frame vertical load is remarkable at frequencies of $1.97 \mathrm{HZ}$ and $3.91 \mathrm{HZ}$, and at high speed, the transmission efficiency at frequencies of $3.91 \mathrm{HZ}$ is remarkable. Based on the dynamic analysis of vehicle system, it is found that the natural frequency of suspension system is $3.83 \mathrm{~Hz}$, which is consistent with the frequency of peak energy of vertical load of frame.

In order to analyze the influence of different frequency components on the structure, we use harmonics of different wavelengths as excitation to analyze. We select the operation speed of 300 $\mathrm{km} / \mathrm{h}$. According to the relevant literature ${ }^{[5]}$, the magnitude is $0.006 \mathrm{~m}$, and the wavelength is increased from 8 to $30 \mathrm{~m}$. The analysis results are as follows.

Table 3 Coefficient of Correlation under Different Harmonic Excitations

\begin{tabular}{|l|l|l|l|l|l|}
\hline wavelength & 4 & 8 & 10 & 20 & 30 \\
\hline frequency & 20.83 & 10.42 & 8.33 & 4.17 & 2.78 \\
\hline correlation & 0.184 & 0.62 & 0.67 & 0.51 & 0.94 \\
\hline
\end{tabular}

As shown in Table 3, the correlation coefficient between them decreases with the decrease of the excitation wavelength, which indicates that the phase difference caused by the transmission of 
different frequency components has a significant effect on the correlation coefficient.

For this reason, the relationship between the vertical load of the frame and the vertical force of wheel and rail is analyzed under different speeds by dividing the low, medium and high frequency sections of the frame, as shown in Table 4.

Table 4 Coefficient of correlation between wheel-rail force and frame load at different speeds and frequencies

\begin{tabular}{|l|l|l|l|}
\hline $\begin{array}{l}\text { frequency } \\
\text { speed( }(\mathrm{km} / \mathrm{h})\end{array}$ & $10 \mathrm{~Hz}$ below & $10 \sim 20 \mathrm{~Hz}$ & $20 \mathrm{~Hz}$ or above \\
\hline 20 & 0.679 & 0.003 & 0.001 \\
\hline 40 & 0.528 & 0.109 & 0.180 \\
\hline 60 & 0.487 & 0.402 & 0.027 \\
\hline 80 & 0.368 & 0.459 & 0.209 \\
\hline 100 & 0.408 & 0.514 & 0.097 \\
\hline 120 & 0.496 & 0.564 & 0.048 \\
\hline 140 & 0.520 & 0.497 & 0.144 \\
\hline 160 & 0.465 & 0.433 & 0.133 \\
\hline 180 & 0.335 & 0.350 & 0.037 \\
\hline 200 & 0.158 & 0.306 & 0.128 \\
\hline 220 & 0.115 & 0.207 & 0.343 \\
\hline 240 & 0.120 & 0.283 & 0.414 \\
\hline 260 & 0.085 & 0.160 & 0.367 \\
\hline 280 & 0.026 & 0.252 & 0.392 \\
\hline 300 & 0.018 & 0.336 & 0.443 \\
\hline
\end{tabular}

From Table 4, it can be seen that the correlation coefficient decreases gradually with the increase of train speed in the frequency range of 0 to $10 \mathrm{~Hz}$; in the frequency range of 10 to $20 \mathrm{~Hz}$, the correlation coefficient increases first and then decreases with the increase of speed; and when the frequency is above $20 \mathrm{~Hz}$, the correlation coefficient increases with speed. Because the influence of phase difference on the correlation coefficient of two variables can not be ignored, it is assumed that the time-domain effective value ratio is used as the transfer coefficient to fit the curve according to the frequency distribution law.

\subsection{Research on Forecasting Method of Wheel-Rail Vertical Force}

From the foregoing, it can be seen that the phase difference between wheel-rail vertical force and frame vertical load caused by the transmission of different frequency components leads to a weak correlation between the two variables, mainly because the transmission efficiency of different frequency components is also different. Therefore, it is proposed that the low frequency range is divided according to the natural frequency of the first suspension system, and the middle and high frequency range is divided according to the peak value distribution of the vertical load of the frame. At the same time, the time-domain effective value ratio of the two frequency ranges is taken as the transfer coefficient. Finally, it is superimposed to complete the curve fitting of the frame load to the wheel-rail force.

\subsection{Fitting Results and Evaluation}

According to the method mentioned above, the vertical force of wheel and rail is fitted by the vertical load of the frame, and the time domain and frequency domain diagrams are drawn respectively. In order to visualize the corresponding relationship between the two, the static load output is subtracted from the result as shown in Figure 7 below. From the drawing results, the fitting degree of the curve is good. 


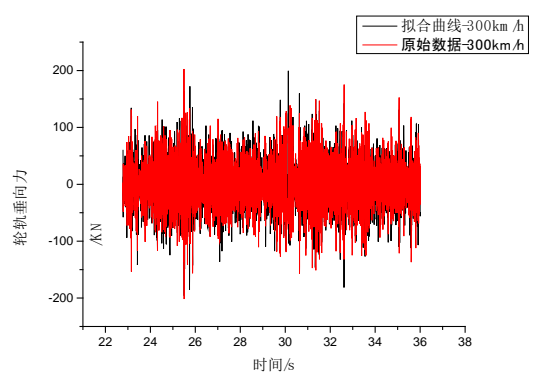

(a) $300 \mathrm{~km} / \mathrm{h}$ wheel/rail vertical force fitting (whole)

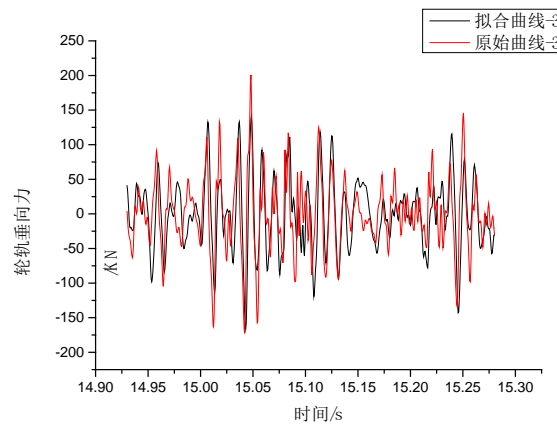

(b) $300 \mathrm{~km} / \mathrm{h}$ wheel/rail vertical force fitting (local)

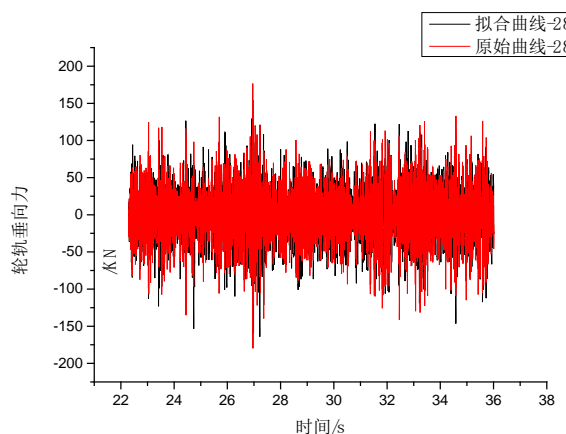

(c) $280 \mathrm{~km} / \mathrm{h}$ wheel/rail vertical force fitting (whole)

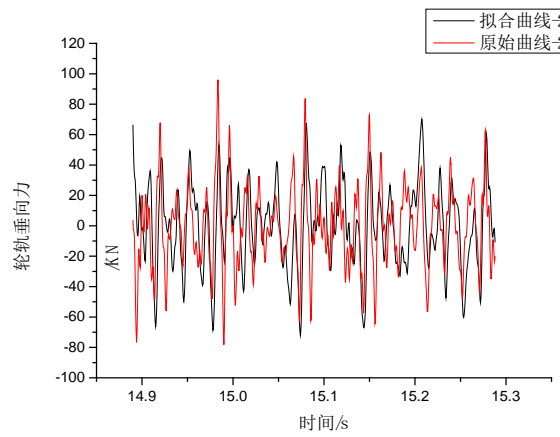

(d) $280 \mathrm{~km} / \mathrm{h}$ wheel/rail vertical force fitting (local)

Fig.6 Fitting Time Domain Diagram of Vertical Force between Medium and High Speed Wheel and Rail

It can be seen from Figure 6 that the above method is used to fit the wheel rail vertical force, and the fitting effect in time domain is good. At the same time, we analyze the fitting result in frequency domain, as shown in Figure 7, the fitting result in frequency domain is also very good. 


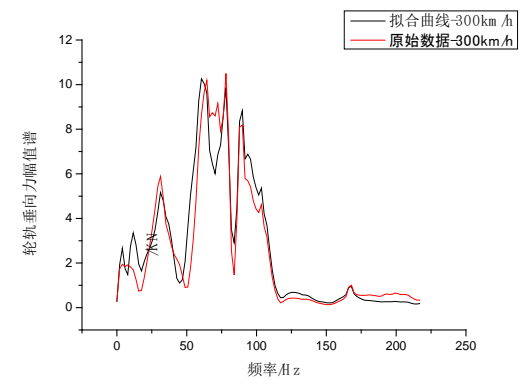

(a) $300 \mathrm{~km} / \mathrm{h}$ wheel/rail vertical force fitting

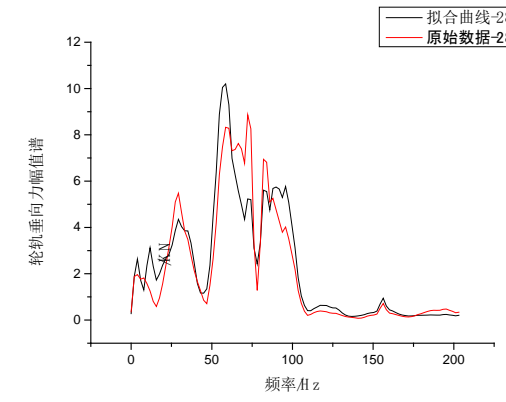

(b) Vertical force fitting of $280 \mathrm{~km} / \mathrm{h}$ wheel/rail

Fig.7 Fitting Frequency Domain Diagram of Vertical Force of High Speed Wheel/Rail

\section{Conclusion}

This paper combines rigid-flexible coupled multi-body dynamics method and finite element method to study the vertical correlation between the frame load and wheel-rail force, and uses the vertical load of the frame to predict the wheel-rail vertical force. The results show that, because the transmission efficiency of different frequencies is different, the intervals can be divided according to the natural frequencies of the suspension system and the frequency peak distribution of the vertical load of the frame. The effective value ratio in time domain is used as the transfer coefficient in each interval. Finally, the intervals are superimposed to complete the vertical load of the frame on the wheel and rail. Force prediction.

\section{References}

[1] Ren Zunsong, Liu Zhiming. Vibration transmission and frequency distribution of high-speed EMUs [J]. Journal of Mechanical Engineering, 2013, 49 (16): 1-7.

[2] Zhang Hongsong, Hu Renxi et al. ANSYS 13.0 finite element analysis from introduction to proficiency [M]. Beijing: Machinery Industry Press, 2011.6:3/296-319.

[3] Miao Bingrong. Fatigue simulation of vehicle body structure based on multi-body dynamics and finite element method [D]. Chengdu: Southwest Jiaotong University, 2006.

[4] Bao Xuehai, Chi Mao-ru, Lu Yaohui, Yang Fei. Research on rigid-flexible hybrid dynamics modeling method of vehicle system based on substructure method [J]. Beijing: Railway locomotive and rolling stock, 2009, 29 (3): 8-11.

[5] Yang Fei, Research on Sensitive Wavelength and Management Value of Long Wave Irregularity of High-speed Railway [D]. Beijing: China Academy of Railway Sciences, 2011:40-46. 